\title{
Exploiting histone deacetylases for cancer therapy: from hematological malignancies to solid tumors
}

\author{
Min Huang \& Meiyu Geng* \\ Division of Antitumor Pharmacology, State Key Laboratory of Drug Research, Shanghai Institute of Materia Medica, Chinese Academy \\ of Sciences, Shanghai 201203, China
}

Received October 9, 2016; accepted October 18, 2016; published online November 23, 2016

Citation: Huang, M., and Geng, M. (2017). Exploiting histone deacetylases for cancer therapy: from hematological malignancies to solid tumors. Sci China Life Sci 60, 94-97. doi: 10.1007/s11427-016-0300-y

Perturbations of the epigenomic landscape, as defined by genome-wide localization of histone post-translational modifications, DNA methylations and chromatin regulatory proteins, have been increasingly appreciated as a common feature of cancer. While epigenetic changes were previously argued as merely a consequence of the cancerous state, recent evidence collectively points to a perception that altered epigenetic states may play a causal role in cancer development (Brien et al., 2016). In fact, cancer genome sequencing studies have shown that genes encoding chromatin regulatory proteins are among the most commonly mutated gene sets in cancer (Garraway and Lander, 2013). In the meanwhile, $25 \%-30 \%$ of the identified cancer driver mutations affect genes encoding chromatin regulatory proteins (Vogelstein et al., 2013) and majority of these somatic mutations are loss-of-function mutations, leading to a reduction or complete loss of chromatin-modifying activities (Garraway and Lander, 2013). Nowadays, it has been widely recognized that changes in epigenetic modifications pattern, alike genetic mutations, play a driving role in cancer formation and persistence. Epigenetic regulators are required for maintaining the expression of a set of key genes in cancer cells, and disturbing this delicate balance may lead to cell catastrophe, thereby connecting "epigenetic vulnerability" to the paradigm of "oncogene addiction" (Brien et al., 2016).

*Correspondence author (email: mygeng@simm.ac.cn)
Moreover, different from genetic mutations caused function loss that is hard to target, the dynamic nature of epigenetic programming is often reversible by targeting distinct epigenetic enzymes.

Global loss of histone acetylation is among the earliest observed epigenetic abnormalities in cancer, and is associated with unfavorable patient outcomes in certain cases (Brien et al., 2016). Histone deacetylases (HDACs) catalyze the removal of acetyl groups from lysine residues in histone amino termini. Intervention of HDACs activity results in global increases in histone acetylation and reverses aberrant epigenetic states in cancer (Figure 1) (Nightingale et al., 2006; Thiagalingam et al., 2003). HDACs in human consist of four classes, class I (HDAC1, HDAC2, HDAC3 and HDAC8), class IIa (HDAC4, HDAC5, HDAC7 and HDAC9), class IIb (HDAC6 and HDAC10), class III (sirtuins 1-7) and class IV (HDAC11). The majority of HDAC inhibitors developed for anticancer therapy inhibits zinc-dependent class I, II and IV HDACs. Among them, vorinostat (suberoylanilide hydroxamic acid, SAHA) was firstly granted Food and Drug Administration (FDA) approval for the treatment of advanced cutaneous $\mathrm{T}$ cell lymphoma in 2006 (Mann et al., 2007). Since this initial success, several HDACs inhibitors including romidepsin, panobinostat, belinostat and chidamide have been proved for the treatment of refractory cutaneous $\mathrm{T}$ cell lymphoma (CTCL), peripheral T-cell lymphoma (PTCL) and multiple 


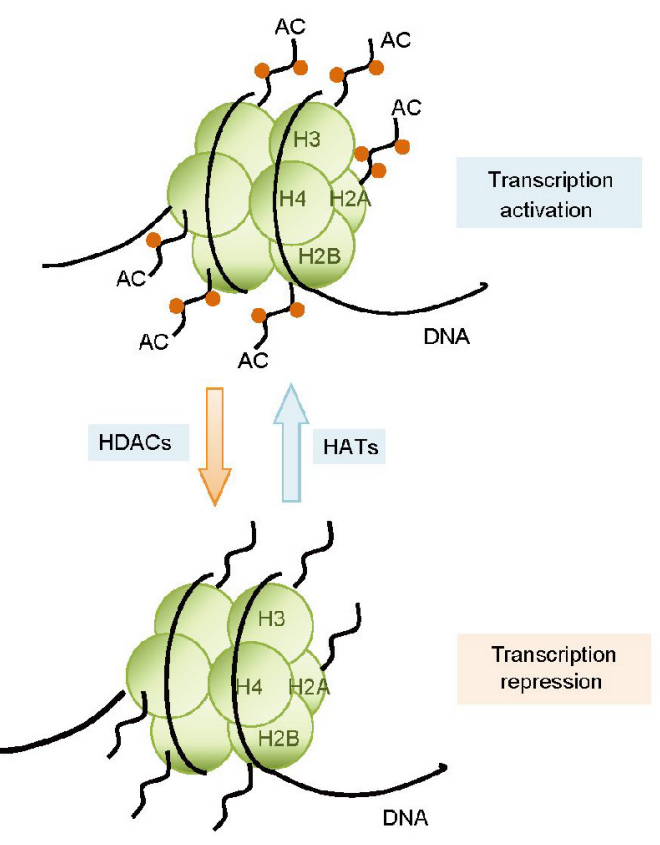

Figure 1 HDACs involved regulation of histone acetylation modifications and transcription activation.

myeloma (Brien et al., 2016). Over 25 HDAC inhibitors are undergoing clinical trials for the potential benefit for different types of cancer at the moment.

Despite the clinical progress of HDAC inhibitors, the detailed mechanism by which HDAC inhibition impedes cancer cell proliferation remains poorly understood. Elevated histone acetylation levels have classically been associated with locally decreased chromatin condensation and increased accessibility for transcription factors (Brien et al., 2016). However, this seems hardly explain the observation that despite widespread hyperacetylation, HDAC inhibition often causes site-specific chromatin remodeling. Recent studies further reveal that specific histone acetylation marks operate within a broader language of epigenetic modifications to orchestrate the functions associated with chromatin, and are dynamically maintained and read by trans-acting "writer", "eraser" and "reader" factors, in which HDACs function as epigenetic erasers (Falkenberg and Johnstone, 2014; Jenuwein and Allis, 2001). This leads to further awareness of the epigenetic language encompassing histone and DNA modifications, and provides mechanisms basis for understanding the anticancer activity of HDAC inhibition. In addition, it is necessary to mention that modulation of the acetylation status of non-histone proteins, particularly transcription factors (Falkenberg and Johnstone, 2014), also contributes to the anticancer effects of HDAC inhibitors (Figure 2).

With several agents approved for clinical use, HDACs inhibitors have been extensively explored in different cancer types, including multiple hematological malignancies and a broad range of solid tumors. At the moment, a major

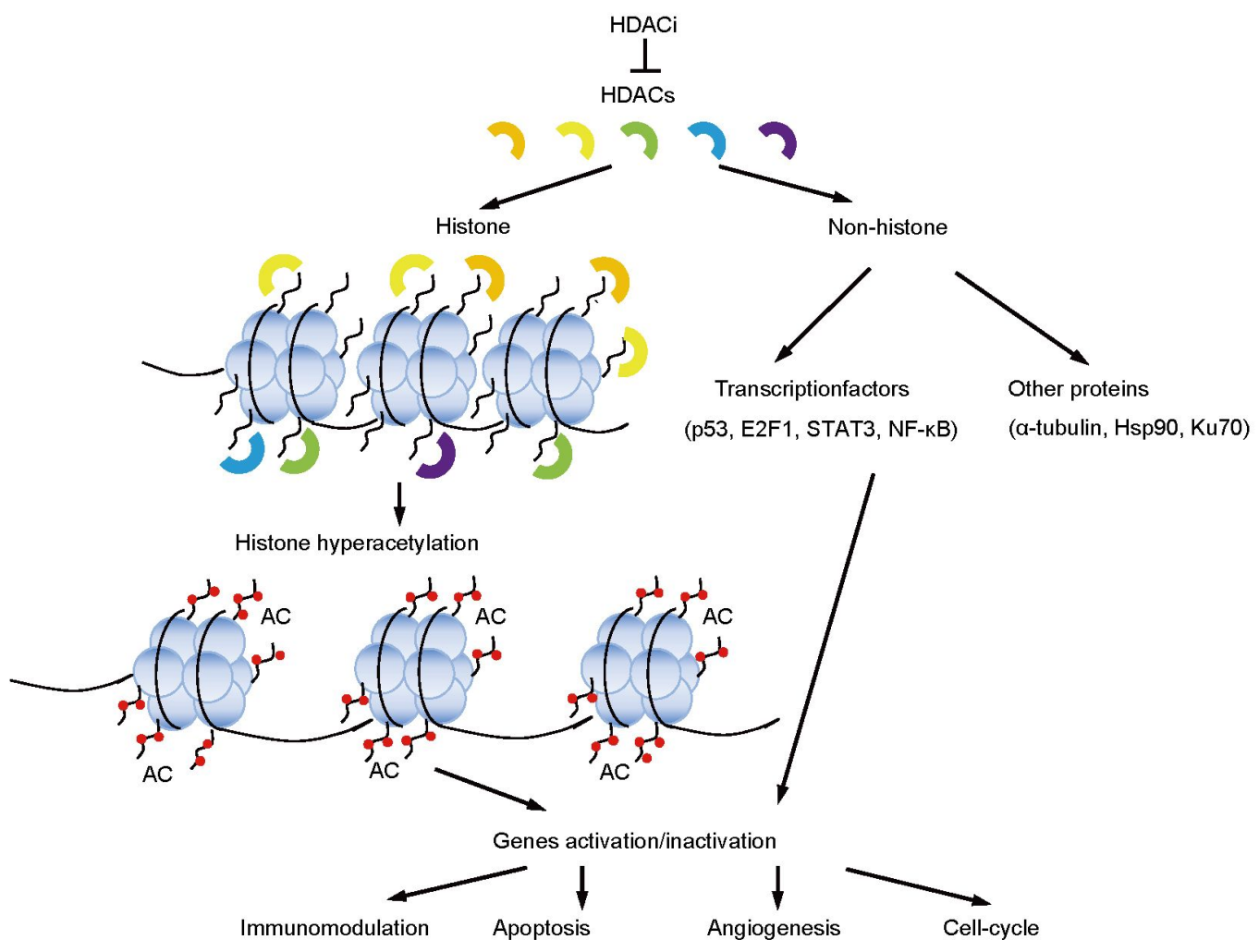

Figure 2 Proposed mechanism of anticancer activity of HDACs inhibitors. 
unanswered question in the field is why HDAC inhibitors have shown a preferential efficacy in subtypes of hematological malignancies (Mercurio et al., 2010). Most of the HDACs inhibitors as single agent failed to show clinical benefits in nearly all types of solid tumors, including breast cancer, renal cancer, prostate cancer, and head and neck cancer (Slingerland et al., 2014). Current studies tend to combine HDACs inhibitors with chemotherapy or other targeted therapies to explore for their utility in solid tumors. Indeed, HDAC inhibitors have been combined with a broad range of agents, both empirically and with rational hypothesis-based approaches. For example, studies that combine DNA methylation and HDAC inhibitors have been initiated, with several clinical trials ongoing examining the utility of these combinations in hematopoietic malignancies and solid tumors (Falkenberg and Johnstone, 2014). Regardless of varying degrees of success in hematopoietic malignancies, it still lacks convincing evidence to conclude the effectiveness in solid tumors. This question has spurred increasing interest in the field, and very recent studies have gained a glimpse of the mechanistic basis in subset of cancer. Zeng et al. has shown that HDAC inhibition increased the expression of leukemia inhibitory factor receptor (LIFR) is a possible mechanism for their therapy failure in breast cancer patients. Drug-induced feedback activation of LIFR-STAT3 anti-apoptotic pathway arises from increased histone acetylation of the LIFR promoter and its transcriptional activation by the acetyllysine reader BRD4. Blocking the resistance mechanism downstream using the approved JAK inhibitor INCB018424 sensitized triple-negative breast cancer (TNBC) cell lines and patient-derived xenografts to vorinostat, suggesting that this combination therapy may improve the clinical utility of HDAC inhibitors in TNBC patients (Zeng et al., 2016). While this molecular basis and derived therapeutic solutions still awaits clinical test, it is predictable that this mechanism may not be applicable to all solid tumors given the heterogeneous property of cancer. Indeed, another study in non-small cell lung cancer (NSCLC) models identifies a different mechanism that involves DNA methyltransferase 1 (DNMT1) induced hypermethylation of insulin-like growth factor 2, which may account for primary and acquired resistance to vorinostat. Co-targeting DNMT1 is shown to improve antitumor efficacy of HDAC inhibitors in NSCLC (Min et al., 2016). These in-depth understanding may promote the more strategic use of HDAC inhibitors in combination with other agents, and expand the scope of their clinical benefits. In parallel, other HDAC involved combinations with therapeutic promise are identified using randomized screening and still lack strong mechanism support. For example, the combination of HDAC with inhibitors for PI3K or BET family proteins were shown as potential therapeutic solutions for MYC-driven medulloblastoma or pancreatic ductal adenocarcinoma respectively
(Mazur et al., 2015; Pei et al., 2016). The therapeutic value revealed by these preclinical combination studies is worthy clinical test and will undoubtedly take prominence in the years to come.

In summary, our knowledge in mechanistic understanding of the cancer epigenome has already delivered solid successes to therapeutically manipulate cancer promoting epigenetic states and cancer-associated gene expression programs. However, our ability to intervene therapeutically in this context is still limited (Brien et al., 2016). A better understanding of the epigenomic alterations that promote oncogenesis and their feedback response to targeted therapies will be necessary to truly harness the therapeutic potential in the manipulation of epigenetic regulation.

Compliance and ethics The author(s) declare that they have no conflict of interest.

Brien, G.L., Valerio, D.G., and Armstrong, S.A. (2016). Exploiting the epigenome to control cancer-promoting gene-expression programs. Cancer Cell 29, 464-476.

Falkenberg, K.J., and Johnstone, R.W. (2014). Histone deacetylases and their inhibitors in cancer, neurological diseases and immune disorders. Nat Rev Drug Discov 13, 673-691.

Garraway, L.A., and Lander, E.S. (2013). Lessons from the cancer genome. Cell 153, 17-37.

Jenuwein, T., and Allis, C.D. (2001). Translating the histone code. Science 293, 1074-1080.

Mann, B.S., Johnson, J.R., Cohen, M.H., Justice, R., and Pazdur, R. (2007). FDA approval summary: vorinostat for treatment of advanced primary cutaneous T-cell lymphoma. Oncologist 12, 1247-1252.

Mazur, P.K., Herner, A., Mello, S.S., Wirth, M., Hausmann, S., SánchezRivera, F.J., Lofgren, S.M., Kuschma, T., Hahn, S.A., Vangala, D., Trajkovic-Arsic, M., Gupta, A., Heid, I., Noël, P.B., Braren, R., Erkan, M., Kleeff, J., Sipos, B., Sayles, L.C., Heikenwalder, M., Heßmann, E., Ellenrieder, V., Esposito, I., Jacks, T., Bradner, J.E., Khatri, P., SweetCordero, E.A., Attardi, L.D., Schmid, R.M., Schneider, G., Sage, J., and Siveke, J.T. (2015). Combined inhibition of BET family proteins and histone deacetylases as a potential epigenetics-based therapy for pancreatic ductal adenocarcinoma. Nat Med 21, 1163-1171.

Mercurio, C., Minucci, S., and Pelicci, P.G. (2010). Histone deacetylases and epigenetic therapies of hematological malignancies. Pharmacol Res $62,18-34$.

Min, H.Y., Lee, S.C., Woo, J.K., Jung, H.J., Park, K.H., Jeong, H.M., Hyun, S.Y., Cho, J., Lee, W., Park, J.E., Kwon, S.J., Lee, H.J., Ni, X., Shin, Y.K., Johnson, F.M., Duvic, M., and Lee, H.Y. (2016). Essential role of DNA methyltransferase 1-mediated transcription of insulin-like growth factor 2 in resistance to histone deacetylase inhibitors. Clin Cancer Res doi: 10.1158/1078-0432.CCR-16-0534.

Nightingale, K.P., O'Neill, L.P., and Turner, B.M. (2006). Histone modifications: signalling receptors and potential elements of a heritable epigenetic code. Curr Opin Genets Dev 16, 125-136.

Pei, Y., Liu, K.W., Wang, J., Garancher, A., Tao, R., Esparza, L.A., Maier, D.L., Udaka, Y.T., Murad, N., Morrissy, S., Seker-Cin, H., Brabetz, S., Qi, L., Kogiso, M., Schubert, S., Olson, J.M., Cho, Y.J., Li, X.N., Crawford, J.R., Levy, M.L., Kool, M., Pfister, S.M., Taylor, M.D., and Wechsler-Reya, R.J. (2016). HDAC and PI3K antagonists cooperate to inhibit growth of MYC-driven medulloblastoma. Cancer Cell 29, 311-323.

Slingerland, M., Guchelaar, H.J., and Gelderblom, H. (2014). Histone deacetylase inhibitors. Anti-Cancer Drugs 25, 140-149.

Thiagalingam, S., Cheng, K.H., Lee, H.J., Mineva, N., Thiagalingam, A., and Ponte, J.F. (2003). Histone deacetylases: unique players in shaping 
the epigenetic histone code. Ann New York Acad Sci 983, 84-100.

Vogelstein, B., Papadopoulos, N., Velculescu, V.E., Zhou, S., Diaz, L.A., and Kinzler, K.W. (2013). Cancer genome landscapes. Science 339, $1546-1558$.

Zeng, H., Qu, J., Jin, N., Xu, J., Lin, C., Chen, Y., Yang, X., He, X., Tang, S.,
Lan, X., Yang, X., Chen, Z., Huang, M., Ding, J., and Geng, M. (2016). Feedback activation of leukemia inhibitory factor receptor limits response to histone deacetylase inhibitors in breast cancer. Cancer Cell 30, 459-473. 